\title{
Sequences of $(\psi, \phi)$-Weakly Contractive Mappings and Stability of Fixed Points in 2-Metric Spaces
}

\author{
S.N. Mishra, Rajendra Pant, And R. Panicker
}

Abstract. The purpose of this paper is to present some new results on the stability of fixed points for certain sequences of weakly contractive mappings, known as $(\psi, \phi)$-weakly contractive mappings over a variable domain in a 2-metric space. The results obtained herein extend certain known results.

\section{INTRODUCTION}

Recently, Barbet and Nachi [5] (see also [4]) obtained some stability results using certain new notions of convergence over a variable domain in a metric space. The above results include the earlier results of Bonsall [6] and Nadler [30]. For some useful references on stability of fixed points, we refer to $[1,3,20,21,22,32,34,35,36,37,38,39]$. These results have been further generalized by Mishra et al.[23, 24, 25, 26, 27, 28, 29] in different settings. In this paper, we obtain a number of stability results in 2-metric spaces for a much wider class of $(\psi, \phi)$-weakly contractive mappings (see Remark 1 below) which include the well known contraction mappings, nonlinear contractions due to Boyd and Wong and weakly contractive mappings (see $[2,8,10,18,33,40,41]$ for details). The results obtained herein thus compliment the results of Barbet and Nachi [5] and extend the result of Mishra et al. [26] to 2-metric spaces. We note that the results so obtained are significant in the sense that 2-metric spaces differ topologically with metric spaces(see Remark 2 below).

Let $(X, d)$ be a metric space and $T: X \rightarrow X$. Then $T$ is called a contraction mapping if there exists a constant $k \in(0,1)$ such that

$$
d(T x, T y) \leq k d(x, y)
$$

for all $x, y \in X$.

2010 Mathematics Subject Classification. Primary: 54H25; $47 \mathrm{H} 10$.

Key words and phrases. Fixed point, stability, 2-metric space, weakly contractive mapping. 
$T: X \rightarrow X$ is called nonlinear contraction [8], if

$$
d(T x, T y) \leq \alpha(d(x, y))
$$

for all $x, y \in X$, where $\alpha:[0, \infty) \rightarrow[0, \infty)$ is upper semicontinuous from the right and $\alpha(t)<t$ for $t>0$. We note that $\alpha(0)=0$.

$T: X \rightarrow X$ is called weakly contractive, if

$$
d(T x, T y) \leq d(x, y)-\phi(d(x, y))
$$

for all $x, y \in X$, where $\phi:[0, \infty) \rightarrow[0, \infty)$ is a continuous and nondecreasing function such that $\phi(t)=0$ if and only if $t=0$.

We note that the condition(3) follows from Tasković [40, 41]. For an earlier work in this direction, we refer to Krasnosel'skii et al. [18] and Dugundji and Granas [10]. Also, that these mappings have been studied by Alber and Guerre-Delabriere [2] and Rhoades [33] as mentioned by Jachymski [17].

In this paper, we shall use the following class of mappings satisfying the so called $(\psi, \phi)$ condition (see for details $[11,7,9]$ ).

$T: X \rightarrow X$ is called $(\psi, \phi)$-weakly contractive if

$$
\psi(d(T x, T y)) \leq \psi(d(x, y))-\phi(d(x, y))
$$

for all $x, y \in X$, where $\psi, \phi:[0, \infty) \rightarrow[0, \infty)$ are both continuous functions such that $\psi(t), \phi(t)>0$ for $t \in(0, \infty)$ and $\psi(0)=0=\phi(0)$. In addition, $\phi$ is non-increasing and $\psi$ is increasing(strictly).

Remark 1. It is interesting to note that if one takes $\phi(t)=(1-k) t$, where $0<k<1$, then (3) reduces to (1). When $\psi(t)=t$, then condition (4) recovers condition (3). In fact, the weakly contractive mappings are also closely related to nonlinear contraction. If $\phi(t)=t-\alpha(t)$, then (3) turns into (2). Therefore

$$
(1) \Rightarrow(2) \Rightarrow(3) \Rightarrow(4)
$$

However, the above implication is not reversible (see [11, Example 2.2]).

This shows the generality of $(\psi, \phi)$-weakly contractive mappings.

\section{2-METRIC SPACES}

We first recall some basics of 2-metric spaces. For details we refer to Gähler [12] and Iséki [13, 14, 15, 16].

Definition 1. Let $X$ be a nonempty set, consisting of at least three points. A 2-metric on $X$ is a real-valued function $\rho$ on $X \times X \times X$ which satisfies the following conditions:

(a) To each pair of distinct points $x, y \in X$ there exists a point $a \in X$ such that $\rho(x, y, a) \neq 0$.

(b) If at least two of $x, y, a$ are equal then $\rho(x, y, a)=0$.

(c) $\rho(x, y, a)=\rho(y, a, x)=\rho(x, a, y)$ for all $x, y, a \in X$. 
(d) $\rho(x, y, a) \leq \rho(x, y, z)+\rho(x, z, a)+\rho(z, y, a)$ for all $x, y, z, a \in X$.

The pair $(X, \rho)$ is called a 2-metric space. $\rho$ is non-negative and it abstracts the properties of the area function for Euclidean triangles in the same manner as a metric abstracts the properties of the length function.

Definition 2. A sequence $\left\{x_{n}\right\}$ in a 2-metric space $(X, \rho)$ is said to be convergent with limit $z \in X$ if $\lim _{n \rightarrow \infty} \rho\left(x_{n}, z, a\right)=0$ for all $a \in X$. Notice that if the sequence $\left\{x_{n}\right\}$ converges to $z$, then $\lim _{n \rightarrow \infty} \rho\left(x_{n}, a, b\right)=\rho(z, a, b)$ for all $a, b \in X$. Further, the sequence $\left\{x_{n}\right\}$ is said to be a Cauchy sequence if $\lim _{m, n \rightarrow \infty} \rho\left(x_{m}, x_{n}, a\right)=0$ for all $a \in X$. If every Cauchy sequence in $X$ is convergent, $(X, \rho)$ is said to be a complete 2 -metric space.

Definition 3. A 2-metric space $(X, \rho)$ is said to be bounded if there is a constant $K$ such that $\rho(a, b, c) \leq K$ for all $a, b, c \in X$.

Remark 2. The following remarks briefly capture some distinct features of topological properties of 2-metric spaces which differ from those of metric spaces.

(i) Given any metric space which consist of more than two points, there always exists a 2-metric compatible with the topology of the space. But the converse is not always true as one can find a 2-metric space which does not have a countable basis associated with one of its arguments (see Gähler [12, Theorem 20 and Example on page 145]).

(ii) A 2-metric $\rho$ is a continuous mapping of each of its three arguments. Generally, we cannot however assert the continuity of $\rho$ in all the three arguments. But if it is continuous in any two arguments simultaneously, then it is continuous in all the three arguments (see Gähler [12, page 123]). So if a 2- metric is continuous in any two arguments, we shall call it continuous.

(iii) In a complete 2-metric space, a convergent sequence need not be Cauchy (see Naidu and Prasad [31, Example 0.1]).

(iv) In a 2-mertic space $(X, \rho)$, every convergent sequence is Cauchy whenever $\rho$ is continuous. However, the converse need not be true (see Naidu and Prasad [31, Example 0.2]).

Now we state the following analogue of conditions (1), (2), (3) and (4) for 2-metric spaces as follows.

Let $(X, \rho)$ be a 2 -metric space and $T: X \rightarrow X$. Then $T$ is called a contraction mapping(or $k$-contraction) if there exists a constant $k \in(0,1)$ such that

$$
\rho(T x, T y, a) \leq k \rho(x, y, a)
$$

for all $x, y, a \in X$. 
It is well known that a contraction mapping on a 2-metric space $X$ has a unique fixed point. Initially, an additional requirement of boundedness was placed on $X$ by Iséki et al.[16] and which was dispensed with subsequently by Rhoades [32] and Lal and Singh [19] independently.

$T: X \rightarrow X$ is called a nonlinear contraction if

$$
\rho(T x, T y, a) \leq \alpha(\rho(x, y, a))
$$

for all $x, y \in X$, where $\alpha:[0, \infty) \rightarrow[0, \infty)$ is upper semicontinuous from the right and $\alpha(t)<t$ for $t>0$. We note that $\alpha(0)=0$.

$T: X \rightarrow X$ is said to be weakly contractive on $X$ if

$$
\rho(T x, T y, a) \leq \rho(x, y, a)-\phi(\rho(x, y, a))
$$

for all $x, y, a \in X$, where $\phi:[0, \infty) \rightarrow[0, \infty)$ is continuous and nondecreasing such that $\phi(t)=0$ if and only if $t=0$.

$T: X \rightarrow X$ is called $(\psi, \phi)$ - weakly contractive if

$$
\psi(\rho(T x, T y, a)) \leq \psi(\rho(x, y, a))-\phi(\rho(x, y, a))
$$

for all $x, y \in X$, where $\psi, \phi:[0, \infty) \rightarrow[0, \infty)$ are both continuous functions such that $\psi(t), \phi(t)>0$ for $t \in(0, \infty)$ and $\psi(0)=0=\phi(0)$. In addition, $\phi$ is non-increasing and $\psi$ is increasing(strictly).

Throughout this paper, let $(X, \rho)$ be a 2-metric space with a continuous 2-metric $\rho$. Let $\mathbb{N}$ be the set of naturals and $\overline{\mathbb{N}}=\mathbb{N} \cup\{\infty\}$.

\section{Stability under ( $\mathrm{G}$ )-CONVERGEnCE}

Definition 4. [27] Let $X$ be a 2-metric space, $\left\{X_{n}\right\}_{n \in \overline{\mathbb{N}}}$ a family of nonempty subsets of $X$ and $\left\{T_{n}: X_{n} \rightarrow X\right\}_{n \in \overline{\mathbb{N}}}$ a family of mappings. Then $T_{\infty}$ is called a (G)-limit of the sequence $\left\{T_{n}\right\}_{n \in \mathbb{N}}$, or equivalently $\left\{T_{n}\right\}_{n \in \overline{\mathbb{N}}}$ satisfies the property $(\mathrm{G})$ if the following condition holds:

(G): $\operatorname{Gr}\left(T_{\infty}\right) \subset \liminf G r\left(T_{n}\right):$ for every $z \in X_{\infty}$, there exists a sequence $\left\{x_{n}\right\}$ in $\prod_{n \in \mathbb{N}} X_{n}$ such that

$\lim _{n \rightarrow \infty} \rho\left(x_{n}, z, a\right)=0$ and $\lim _{n \rightarrow \infty} \rho\left(T_{n} x_{n}, T_{\infty} z, a\right)=0 \quad$ for all $a \in X$,

where $\operatorname{Gr}(T)$ denotes the graph of $T$.

The following proposition extends a result of Barbet and Nachi [5, Proposition 1] (see also Mishra et al.[26]) for $(\psi, \phi)$-weakly contractive mappings to a 2-metric space.

Proposition 1. Let $X$ be a 2-metric space, $\left\{X_{n}\right\}_{n \in \overline{\mathbb{N}}}$ a family of nonempty subsets of $X$ and $\left\{T_{n}: X_{n} \rightarrow X\right\}_{n \in \mathbb{N}}$ a sequence of $(\psi, \phi)$-weakly contractive mappings. If $T_{\infty}: X_{\infty} \rightarrow X$ is a $(G)$-limit of $\left\{T_{n}\right\}$, then $T_{\infty}$ is unique. 
Proof. Assume that $T_{\infty}: X_{\infty} \rightarrow X$ and $T_{\infty}^{*}: X_{\infty} \rightarrow X$ are (G)-limit mappings of the sequence $\left\{T_{n}\right\}$. Hence for any point $x \in X_{\infty}$, there exist two sequences $\left\{x_{n}\right\}$ and $\left\{y_{n}\right\}$ in $\prod_{n \in \mathbb{N}} X_{n}$ converging to $x$ such that $\left\{T_{n} x_{n}\right\}$ and $\left\{T_{n} y_{n}\right\}$ converge to $T_{\infty}$ and $T_{\infty}^{*}$ respectively. Therefore

$$
\lim _{n \rightarrow \infty} \rho\left(T_{n} x_{n}, T_{\infty} x, a\right)=0, \lim _{n \rightarrow \infty} \rho\left(T_{n} y_{n}, T_{\infty}^{*} x, a\right)=0 \text { for all } a \in X .
$$

Since $T_{n}$ is $(\psi, \phi)$-weakly contractive for each $n \in \mathbb{N}$,

$$
\psi\left(\rho\left(T_{n} x_{n}, T_{n} y_{n}, a\right)\right) \leq \psi\left(\rho\left(x_{n}, y_{n}, a\right)\right)-\phi\left(\rho\left(x_{n}, y_{n}, a\right)\right)
$$

which implies that

$$
\psi\left(\rho\left(T_{n} x_{n}, T_{n} y_{n}, a\right)\right) \leq \psi\left(\rho\left(x_{n}, y_{n}, a\right)\right) .
$$

As $\psi$ is increasing, from the above inequality we have

$$
\rho\left(T_{n} x_{n}, T_{n} y_{n}, a\right) \leq \rho\left(x_{n}, y_{n}, a\right) .
$$

By the triangular area inequality and condition (9), for all $n \in \mathbb{N}$ and for any $a \in X$, we have

$$
\begin{aligned}
& \quad \rho\left(T_{\infty} x, T_{\infty}^{*} x, a\right) \\
& \leq \rho\left(T_{\infty} x, T_{\infty}^{*} x, T_{n} x_{n}\right)+\rho\left(T_{\infty} x, T_{n} x_{n}, a\right)+\rho\left(T_{n} x_{n}, T_{\infty}^{*} x, a\right) \\
& \leq \rho\left(T_{\infty} x, T_{\infty}^{*} x, T_{n} x_{n}\right)+\rho\left(T_{\infty} x, T_{n} x_{n}, a\right)+\rho\left(T_{n} x_{n}, T_{\infty}^{*} x, T_{n} y_{n}\right) \\
& \quad+\rho\left(T_{n} x_{n}, T_{n} y_{n}, a\right)+\rho\left(T_{n} y_{n}, T_{\infty}^{*} x, a\right) \\
& \leq \rho\left(T_{\infty} x, T_{\infty}^{*} x, T_{n} x_{n}\right)+\rho\left(T_{\infty} x, T_{n} x_{n}, a\right)+\rho\left(T_{n} x_{n}, T_{\infty}^{*} x, T_{n} y_{n}\right) \\
& \quad+\rho\left(x_{n}, y_{n}, a\right)+\rho\left(T_{n} y_{n}, T_{\infty}^{*} x, a\right) \rightarrow 0 \text { as } n \rightarrow \infty .
\end{aligned}
$$

Hence we deduce that $\lim _{n \rightarrow \infty} \rho\left(T_{\infty} x, T_{\infty}^{*} x, a\right)=0$ and the unicity of the limit is established.

When $\psi(t)=t$ and $\phi(t)=(1-k) t$ and $k \in(0,1)$ in the above proposition, we get the following result.

Corollary 1 ([27, Proposition 2.2]). Let $X$ be a 2-metric space, $\left\{X_{n}\right\}_{n \in \overline{\mathbb{N}}}$ a family of nonempty subsets of $X$ and $\left\{T_{n}: X_{n} \rightarrow X\right\}_{n \in \mathbb{N}}$ a sequence of $k$-contraction mappings. If $T_{\infty}: X_{\infty} \rightarrow X$ is a $(G)$-limit of $\left\{T_{n}\right\}$, then $T_{\infty}$ is unique.

When $\psi(t)=t$ and $\phi(t)=t-\alpha(t)$ in the above proposition, the following result is obtained.

Corollary 2. [25, Proposition 3.3] Corollary 1 with $k$-contraction replaced by nonlinear contraction.

The following theorem is our first stability result. 
Theorem 1. Let $X$ be a 2-metric space, $\left\{X_{n}\right\}_{n \in \overline{\mathbb{N}}}$ a family of nonempty subsets of $X$ and $\left\{T_{n}: X_{n} \rightarrow X\right\}_{n \in \overline{\mathbb{N}}}$ a family of mappings satisfying the property $(G)$ such that for all $n \in \mathbb{N}, T_{n}$ is a $(\psi, \phi)$-weakly contractive mapping where $\psi$ is increasing and $\phi$ is non-increasing. If for all $n \in \overline{\mathbb{N}}, x_{n}$ is a fixed point of $T_{n}$ then the sequence $\left\{x_{n}\right\}_{n \in \mathbb{N}}$ converges to $x_{\infty}$.

Proof. Let $x_{n}$ be a fixed point of $T_{n}$ for each $n \in \overline{\mathbb{N}}$. Since the property $(\mathrm{G})$ holds and $x_{\infty} \in X_{\infty}$, there exists a sequence $\left\{y_{n}\right\}$ in $\prod_{n \in \mathbb{N}} X_{n}$ such that

$$
\lim _{n \rightarrow \infty} \rho\left(y_{n}, x_{\infty}, a\right)=0 \text { and } \lim _{n \rightarrow \infty} \rho\left(T_{n} y_{n}, T_{\infty} x_{\infty}, a\right)=0 \text { for all } a \in X .
$$

We have

$$
\begin{aligned}
\psi\left(\rho\left(x_{n}, x_{\infty}, a\right)\right) & =\psi\left(\rho\left(T_{n} x_{n}, T_{\infty} x_{\infty}, a\right)\right) \\
& \leq \psi\left(\rho\left(T_{n} x_{n}, T_{\infty} x_{\infty}, T_{n} y_{n}\right)+\rho\left(T_{n} x_{n}, T_{n} y_{n}, a\right)+\rho\left(T_{n} y_{n}, T_{\infty} x_{\infty}, a\right)\right) .
\end{aligned}
$$

Making $n \rightarrow \infty$ in the above inequality and using the continuity of $\psi$ and $\phi$ and the fact that $\psi$ is increasing and $\phi$ is non-increasing, we obtain

$$
\begin{aligned}
\lim _{n \rightarrow \infty} \psi\left(\rho\left(x_{n}, x_{\infty}, a\right)\right) \leq & \lim _{n \rightarrow \infty} \psi\left(\rho\left(T_{n} x_{n}, T_{\infty} x_{\infty}, T_{n} y_{n}\right)\right. \\
& \left.+\rho\left(T_{n} x_{n}, T_{n} y_{n}, a\right)+\rho\left(T_{n} y_{n}, T_{\infty} x_{\infty}, a\right)\right) \\
= & \lim _{n \rightarrow \infty} \psi\left(\rho\left(T_{n} x_{n}, T_{n} y_{n}, a\right)\right) \\
\leq & \lim _{n \rightarrow \infty}\left[\psi\left(\rho\left(x_{n}, y_{n}, a\right)\right)-\phi\left(\rho\left(x_{n}, y_{n}, a\right)\right)\right] \\
\leq & \lim _{n \rightarrow \infty}\left[\psi\left(\rho\left(x_{n}, y_{n}, x_{\infty}\right)+\rho\left(x_{n}, x_{\infty}, a\right)+\rho\left(x_{\infty}, y_{n}, a\right)\right)\right. \\
& \left.\quad-\phi\left(\rho\left(x_{n}, y_{n}, x_{\infty}\right)+\rho\left(x_{n}, x_{\infty}, a\right)+\rho\left(x_{\infty}, y_{n}, a\right)\right)\right] \\
= & \lim _{n \rightarrow \infty} \psi\left(\rho\left(x_{n}, x_{\infty}, a\right)\right)-\lim _{n \rightarrow \infty} \phi\left(\rho\left(x_{n}, x_{\infty}, a\right)\right),
\end{aligned}
$$

which implies that

$$
\lim _{n \rightarrow \infty} \phi\left(\rho\left(x_{n}, x_{\infty}, a\right)\right) \leq 0 .
$$

By the property of $\phi$, we get $\lim _{n \rightarrow \infty} \rho\left(x_{n}, x_{\infty}, a\right)=0$ and hence the conclusion.

Corollary 3 ([27, Theorem 2.3]). Let $X$ be a 2-metric space, $\left\{X_{n}\right\}_{n \in \overline{\mathbb{N}}}$ a family of nonempty subsets of $X$ and $\left\{T_{n}: X_{n} \rightarrow X\right\}_{n \in \overline{\mathbb{N}}}$ a family of mappings satisfying the property $(G)$ such that, for all $n \in \mathbb{N}, T_{n}$ is a $k$ contraction. If for all $n \in \overline{\mathbb{N}}, x_{n}$ is a fixed point of $T_{n}$ then the sequence $\left\{x_{n}\right\}_{n \in \mathbb{N}}$ converges to $x_{\infty}$.

Proof. It comes from Theorem 1 when $\psi(t)=t$ and $\phi(t)=(1-k) t$ and $k \in(0,1)$.

Corollary 4 ([25, Theorem 3.5]). Corollary 3 with $k$-contraction replaced by nonlinear contraction. 
The existence of a fixed point for a $(\mathrm{G})$-limit mapping is characterized by the following result when it is a $(\psi, \phi)$-weakly contractive mapping.

Theorem 2. Let $X$ be a 2-metric space, $\left\{X_{n}\right\}_{n \in \overline{\mathbb{N}}}$ a family of nonempty subsets of $X$ and $\left\{T_{n}: X_{n} \rightarrow X\right\}_{n \in \overline{\mathbb{N}}}$ a family of mappings satisfying the property $(G)$ and such that for any $n \in \mathbb{N}, T_{n}$ is a $(\psi, \phi)$-weakly contractive mapping. Assume that, for any $n \in \mathbb{N}, x_{n}$ is a fixed point of $T_{n}$. Then

$T_{\infty}$ admits a fixed point $\Leftrightarrow\left\{x_{n}\right\}$ converges and $\lim x_{n} \in X_{\infty}$

$\Leftrightarrow\left\{x_{n}\right\}$ admits a subsequence converging to a point of $X_{\infty}$.

Proof. The necessary part is already proved in Theorem 1. To prove the sufficiency, let $\left\{x_{n_{j}}\right\}$ be a subsequence of $\left\{x_{n}\right\}$ such that $\lim _{j \rightarrow \infty} x_{n_{j}}=x_{\infty} \in$ $X_{\infty}$. By the property $(\mathrm{G})$, there exists a sequence $\left\{y_{n}\right\}$ in $\prod_{n \in \mathbb{N}} X_{n}$ such that

$$
\lim _{n \rightarrow \infty} \rho\left(y_{n}, x_{\infty}, a\right)=0 \text { and } \lim _{n \rightarrow \infty} \rho\left(T_{n} y_{n}, T_{\infty} x_{\infty}, a\right)=0 \text { for all } a \in X .
$$

Hence for any $a \in X$ and $n \in \mathbb{N}$, we have

$$
\begin{aligned}
& \quad \rho\left(x_{\infty}, T_{\infty} x_{\infty}, a\right) \\
& \leq \rho\left(x_{\infty}, x_{n_{j}}, a\right)+\rho\left(T_{n_{j}} x_{n_{j}}, T_{\infty} x_{\infty}, a\right)+\rho\left(x_{\infty}, T_{\infty} x_{\infty}, T_{n_{j}} x_{n_{j}}\right) \\
& \leq \rho\left(x_{\infty}, x_{n_{j}}, a\right)+\rho\left(T_{n_{j}} x_{n_{j}}, T_{\infty} x_{\infty}, T_{n_{j}} y_{n_{j}}\right)+\rho\left(T_{n_{j}} x_{n_{j}}, T_{n_{j}} y_{n_{j}}, a\right) \\
& \quad+\rho\left(T_{n_{j}} y_{n_{j}}, T_{\infty} x_{\infty}, a\right)+\rho\left(x_{\infty}, T_{\infty} x_{\infty}, T_{n_{j}} x_{n_{j}}\right) \\
& \quad \leq \rho\left(x_{\infty}, x_{n_{j}}, a\right)+\rho\left(T_{n_{j}} x_{n_{j}}, T_{\infty} x_{\infty}, T_{n_{j}} y_{n_{j}}\right)+\rho\left(x_{n_{j}}, y_{n_{j}}, a\right) \\
& \quad+\rho\left(T_{n_{j}} y_{n_{j}}, T_{\infty} x_{\infty}, a\right)+\rho\left(x_{\infty}, T_{\infty} x_{\infty}, T_{n_{j}} x_{n_{j}}\right) \text { by condition }(9) .
\end{aligned}
$$

The right hand side of the above expression tends to zero as $j \rightarrow \infty$ and hence $T_{\infty} x_{\infty}=x_{\infty}$, proving that $x_{\infty}$ is a fixed point of $T_{\infty}$.

Corollary 5 ([25, Theorem 3.8]). Let $X$ be a 2-metric space, $\left\{X_{n}\right\}_{n \in \overline{\mathbb{N}}} a$ family of nonempty subsets of $X$ and $\left\{T_{n}: X_{n} \rightarrow X\right\}_{n \in \overline{\mathbb{N}}}$ a family of mappings satisfying the property $(G)$ and such that for any $n \in \mathbb{N}, T_{n}$ is a nonlinear contraction. Assume that for any $n \in \mathbb{N}, x_{n}$ is a fixed point of $T_{n}$. Then

$T_{\infty}$ admits a fixed point $\Leftrightarrow\left\{x_{n}\right\}$ converges and $\lim x_{n} \in X_{\infty}$

$\Leftrightarrow\left\{x_{n}\right\}$ admits a subsequence converging to a point of $X_{\infty}$.

Remark 3. Under the assumptions of Theorem 2, and if

(i): $\liminf X_{n} \subset X_{\infty}$ (i.e., the limit of any convergent sequence $\left\{z_{n}\right\}$ in $\prod_{n \in \mathbb{N}} X_{n}$ is in $\left.X_{\infty}\right)$, then

$T_{\infty}$ admits a fixed point $\Leftrightarrow\left\{x_{n}\right\}$ converges. 
(ii): $\lim \sup X_{n} \subset X_{\infty}$ (i.e., the cluster point of any sequence $\left\{z_{n}\right\}$ in $\prod_{n \in \mathbb{N}} X_{n}$ is in $\left.X_{\infty}\right)$ then

$T_{\infty}$ admits a fixed point $\Leftrightarrow\left\{x_{n}\right\}$ admits a convergent subsequence.

The following proposition provides a sufficient condition under which a $(\mathrm{G})$-limit of a sequence of $(\psi, \phi)$-weakly contractive mappings is again $(\psi, \phi)$ weakly contractive.

Proposition 2. Let $X$ be a 2-metric space, $\left\{X_{n}\right\}_{n \in \overline{\mathbb{N}}}$ a family of nonempty subsets of $X$ and $\left\{T_{n}: X_{n} \rightarrow X\right\}_{n \in \overline{\mathbb{N}}}$ a family of mappings satisfying the property $(G)$ and such that for any $n \in \mathbb{N}, T_{n}$ is a $(\psi, \phi)$-weakly contractive mapping. Then $T_{\infty}$ is $(\psi, \phi)$-weakly contractive.

Proof. Given two points $x$ and $y$ in $X_{\infty}$, by the property $(\mathrm{G})$ there exist two sequences $\left\{x_{n}\right\}$ and $\left\{y_{n}\right\}$ in $\prod_{n \in \mathbb{N}} X_{n}$ converging respectively to $x$ and $y$ such that the sequences $\left\{T_{n} x_{n}\right\}$ and $\left\{T_{n} y_{n}\right\}$ converge respectively to $T_{\infty} x$ and $T_{\infty} y$. For any $n \in \mathbb{N}$ and $a \in X$,

$$
\begin{aligned}
& \psi\left(\rho\left(T_{\infty} x, T_{\infty} y, a\right)\right) \\
\leq & \psi\left(\rho\left(T_{\infty} x, T_{\infty} y, T_{n} x_{n}\right)+\rho\left(T_{\infty} x, T_{n} x_{n}, a\right)+\rho\left(T_{n} x_{n}, T_{\infty} y, a\right)\right) \\
\leq & \psi\left(\rho\left(T_{\infty} x, T_{\infty} y, T_{n} x_{n}\right)+\rho\left(T_{\infty} x, T_{n} x_{n}, a\right)+\rho\left(T_{n} x_{n}, T_{\infty} y, T_{n} y_{n}\right)\right. \\
& \left.\quad+\rho\left(T_{n} x_{n}, T_{n} y_{n}, a\right)+\rho\left(T_{n} y_{n}, T_{\infty} y, a\right)\right) .
\end{aligned}
$$

Letting $n \rightarrow \infty$, and using the continuity of both $\psi$ and $\phi$ we have

$$
\begin{aligned}
\psi\left(\rho\left(T_{\infty} x, T_{\infty} y, a\right)\right) & \leq \lim _{n \rightarrow \infty} \psi\left(\rho\left(T_{n} x_{n}, T_{n} y_{n}, a\right)\right) \\
& \leq \lim _{n \rightarrow \infty}\left[\psi\left(\rho\left(x_{n}, y_{n}, a\right)\right)-\phi\left(\rho\left(x_{n}, y_{n}, a\right)\right)\right] .
\end{aligned}
$$

Hence we conclude that $\psi\left(\rho\left(T_{\infty} x, T_{\infty} y, a\right)\right) \leq \psi(\rho(x, y, a))-\phi(\rho(x, y, a))$.

Corollary 6. Let $X$ be a 2-metric space, $\left\{X_{n}\right\}_{n \in \overline{\mathbb{N}}}$ a family of nonempty subsets of $X$ and $\left\{T_{n}: X_{n} \rightarrow X\right\}_{n \in \overline{\mathbb{N}}}$ a family of mappings satisfying the property $(G)$ and such that for any $n \in \mathbb{N}, T_{n}$ is a $k$-contraction from $X_{n}$ to $X$. Then $T_{\infty}$ is a k-contraction.

Proof. This comes from Proposition 2 when $\psi(t)=t$ and $\phi(t)=(1-k) t$ and $k \in(0,1)$.

Corollary 7 ([25, Proposition 3.10]). Corollary 6 with $k$-contraction replaced by nonlinear contraction.

Under a compactness assumption, the existence of a fixed point of the (G)-limit mapping can be obtained from the existence of fixed points of the $(\psi, \phi)$-weakly contractive mappings $T_{n}$. 
Theorem 3. Let $\left\{X_{n}\right\}_{n \in \overline{\mathbb{N}}}$ be a family of nonempty subsets of a 2-metric space $X$ and $\left\{T_{n}: X_{n} \rightarrow X\right\}_{n \in \overline{\mathbb{N}}}$ a family of mappings satisfying the property $(G)$ and such that, for any $n \in \mathbb{N}, T_{n}$ is a $(\psi, \phi)$-weakly contractive mapping where $\psi$ is increasing and $\phi$ is non-increasing. Assume that $\lim \sup X_{n} \subset$ $X_{\infty}$ and $\bigcup_{n \in \mathbb{N}} X_{n}$ is relatively compact. If for any $n \in \mathbb{N}, T_{n}$ admits a fixed point $x_{n}$, then the $(G)$-limit mapping $T_{\infty}$ admits a fixed point $x_{\infty}$ and the sequence $\left\{x_{n}\right\}_{n \in \mathbb{N}}$ converges to $x_{\infty}$.

Proof. Let $x_{n}$ be the fixed point of $T_{n}$ for $n \in \mathbb{N}$. From compactness condition, there exists a convergent subsequence $\left\{x_{n_{j}}\right\}$ of $\left\{x_{n}\right\}$. Now by Remark $3, T_{\infty}$ admits a fixed point $x_{\infty}$ and by Theorem 1 , the sequence $\left\{x_{n}\right\}$ converges to $x_{\infty}$.

Corollary 8 ([27, Theorem 2.10]). Let $\left\{X_{n}\right\}_{n \in \overline{\mathbb{N}}}$ be a family of nonempty subsets of a 2-metric space $X$ and $\left\{T_{n}: X_{n} \rightarrow X\right\}_{n \in \overline{\mathbb{N}}}$ a family of mappings satisfying the property $(G)$ and such that, for any $n \in \mathbb{N}, T_{n}$ is a $k$-contraction. Assume that $\lim \sup X_{n} \subset X_{\infty}$ and $\bigcup_{n \in \mathbb{N}} X_{n}$ is relatively compact. If for any $n \in \mathbb{N}, T_{n}$ admits a fixed point $x_{n}$, then the (G)-limit mapping $T_{\infty}$ admits a fixed point $x_{\infty}$ and the sequence $\left\{x_{n}\right\}_{n \in \mathbb{N}}$ converges to $x_{\infty}$.

Proof. This comes from Theorem 3, when $\psi(t)=t$ and $\phi(t)=(1-k) t$ and $k \in(0,1)$.

Corollary 9 ([25, Theorem 3.12]). Corollary 8 with $k$-contraction replaced by nonlinear contraction.

Proof. This comes from Theorem 3, when $\psi(t)=t$ and $\phi(t)=t-\alpha(t)$.

The following notion of convergence is weaker than $(\mathrm{G})$-convergence and has been studied in [27].

Definition 5. [27] Let $X$ be a 2-metric space, $\left\{X_{n}\right\}_{n \in \overline{\mathbb{N}}}$ a family of nonempty subsets of $X$ and $\left\{T_{n}: X_{n} \rightarrow X\right\}_{n \in \overline{\mathbb{N}}}$ a family of mappings. Then $T_{\infty}$ is called a $\left(\mathrm{G}^{-}\right)$limit of the sequence $\left\{T_{n}\right\}_{n \in \mathbb{N}}$, or equivalently $\left\{T_{n}\right\}_{n \in \overline{\mathbb{N}}}$ satisfies the property $\left(\mathrm{G}^{-}\right)$, if the following condition holds:

$\left(\mathbf{G}^{-}\right): G r\left(T_{\infty}\right) \subset \limsup G r\left(T_{n}\right):$ for all $z \in X_{\infty}$, there exists a sequence $\left\{x_{n}\right\}_{n \in \mathbb{N}}$ in $\prod_{n \in \mathbb{N}} X_{n}$, which has a subsequence $\left\{x_{n_{j}}\right\}$ such that

$$
\lim _{j \rightarrow \infty} \rho\left(x_{n_{j}}, z, a\right)=0 \text { and } \lim _{j \rightarrow \infty} \rho\left(T_{n_{j}} x_{n_{j}}, T_{\infty} z, a\right)=0 \text {, for all } a \in X .
$$

The following result establishes that a fixed point of a $\left(\mathrm{G}^{-}\right)$-limit mapping is a cluster point of the sequence of fixed points associated with $\left\{T_{n}\right\}$. 
Theorem 4. Let $\left\{X_{n}\right\}$ be a family of nonempty subsets of a 2-metric space $X$ and $\left\{T_{n}: X_{n} \rightarrow X\right\}_{n \in \overline{\mathbb{N}}}$ a family of $(\psi, \phi)$-weakly contractive mappings satisfying the property $\left(G^{-}\right)$, where $\psi$ is increasing and $\phi$ is non-increasing. If for any $n \in \overline{\mathbb{N}}, x_{n}$ is a fixed point of $T_{n}$ then $x_{\infty}$ is a cluster point of the sequence $\left\{x_{n}\right\}_{n \in \mathbb{N}}$.

Proof. By the property $\left(\mathrm{G}^{-}\right)$, there exists a sequence $\left\{y_{n}\right\}$ in $\prod_{n \in \mathbb{N}} X_{n}$ which has a subsequence $\left\{y_{n_{j}}\right\}$ such that

$$
\lim _{j \rightarrow \infty} \rho\left(y_{n_{j}}, x_{\infty}, a\right)=0 \text { and } \lim _{j \rightarrow \infty} \rho\left(T_{n_{j}} y_{n_{j}}, T_{\infty} x_{\infty}, a\right)=0 \text { for all } a \in X .
$$

By the triangular area inequality, we have

$$
\begin{aligned}
\rho\left(x_{n_{j}}, x_{\infty}, a\right) \leq & \rho\left(T_{n_{j}} x_{n_{j}}, T_{\infty} x_{\infty}, T_{n_{j}} y_{n_{j}}\right) \\
& +\rho\left(T_{n_{j}} x_{n_{j}}, T_{n_{j}} y_{n_{j}}, a\right)+\rho\left(T_{n_{j}} y_{n_{j}}, T_{\infty} x_{\infty}, a\right) .
\end{aligned}
$$

Since $\psi$ is increasing,

$$
\begin{aligned}
\psi\left(\rho\left(x_{n_{j}}, x_{\infty}, a\right)\right) \leq & \psi\left(\rho\left(T_{n_{j}} x_{n_{j}}, T_{\infty} x_{\infty}, T_{n_{j}} y_{n_{j}}\right)\right. \\
& \left.+\rho\left(T_{n_{j}} x_{n_{j}}, T_{n_{j}} y_{n_{j}}, a\right)+\rho\left(T_{n_{j}} y_{n_{j}}, T_{\infty} x_{\infty}, a\right)\right) .
\end{aligned}
$$

Now using the properties of $\psi$ and $\phi$ and making $j \rightarrow \infty$, we get

$$
\begin{aligned}
& \lim _{j \rightarrow \infty} \psi\left(\rho\left(x_{n_{j}}, x_{\infty}, a\right)\right) \\
\leq & \lim _{j \rightarrow \infty} \psi\left(\rho\left(T_{n_{j}} x_{n_{j}}, T_{n_{j}} y_{n_{j}}, a\right)\right) \\
\leq & \lim _{j \rightarrow \infty}\left[\psi\left(\rho\left(x_{n_{j}}, y_{n_{j}}, a\right)\right)-\phi\left(\rho\left(x_{n_{j}}, y_{n_{j}}, a\right)\right)\right] \\
\leq & \lim _{j \rightarrow \infty}\left[\psi\left(\rho\left(x_{n_{j}}, y_{n_{j}}, x_{\infty}\right)+\rho\left(x_{n_{j}}, x_{\infty}, a\right)+\rho\left(x_{\infty}, y_{n_{j}}, a\right)\right)\right. \\
& \left.\quad-\phi\left(\rho\left(x_{n_{j}}, y_{n_{j}}, x_{\infty}\right)+\rho\left(x_{n_{j}}, x_{\infty}, a\right)+\rho\left(x_{\infty}, y_{n_{j}}, a\right)\right)\right] \\
= & \lim _{j \rightarrow \infty} \psi\left(\rho\left(x_{n_{j}}, x_{\infty}, a\right)\right)-\lim _{j \rightarrow \infty} \phi\left(\rho\left(x_{n_{j}}, x_{\infty}, a\right)\right) .
\end{aligned}
$$

Hence

$$
\lim _{j \rightarrow \infty} \phi\left(\rho\left(x_{n_{j}}, x_{\infty}, a\right)\right) \leq 0 .
$$

By the property of $\phi$, we deduce that

$$
\lim _{j \rightarrow \infty} \rho\left(x_{n_{j}}, x_{\infty}, a\right)=0 .
$$

Thus $\left\{x_{n_{j}}\right\}$ converges to $x_{\infty}$, the fixed point of $T_{\infty}$.

Corollary 10 ([27, Theorem 2.12]). Let $\left\{X_{n}\right\}$ be a family of nonempty subsets of a 2-metric space $X$ and $\left\{T_{n}: X_{n} \rightarrow X\right\}_{n \in \overline{\mathbb{N}}}$ a family of $k$-contraction mappings satisfying the property $\left(G^{-}\right)$. If for any $n \in \mathbb{N}, x_{n}$ is a fixed point of $T_{n}$, then $x_{\infty}$ is a cluster point of the sequence $\left\{x_{n}\right\}_{n \in \mathbb{N}}$. 
Proof. This comes from Theorem 4, when $\psi(t)=t$ and $\phi(t)=(1-k) t$ and $k \in(0,1)$.

Corollary 11 ([25, Theorem 3.15]). Corollary 10 with $k$-contraction replaced by nonlinear contraction.

\section{Stability under (H)-CONVERGence}

Definition 6. [27] Let $X$ be a 2-metric space, $\left\{X_{n}\right\}_{n \in \overline{\mathbb{N}}}$ a family of nonempty subsets of $X$ and $\left\{T_{n}: X_{n} \rightarrow X\right\}_{n \in \overline{\mathbb{N}}}$ a family of mappings. Then $T_{\infty}$ is called an (H)-limit of the sequence $\left\{T_{n}\right\}_{n \in \mathbb{N}}$ or, equivalently $\left\{T_{n}\right\}_{n \in \overline{\mathbb{N}}}$ satisfies the property $(\mathrm{H})$ if the following condition holds:

(H): For all sequences $\left\{x_{n}\right\}$ in $\prod_{n \in \mathbb{N}} X_{n}$, there exists a sequence $\left\{y_{n}\right\}$ in $X_{\infty}$ such that

$$
\lim _{n \rightarrow \infty} \rho\left(x_{n}, y_{n}, a\right)=0 \text { and } \lim _{n \rightarrow \infty} \rho\left(T_{n} x_{n}, T_{\infty} y_{n}, a\right)=0 \text { for all } a \in X .
$$

The following theorem is our second stability result using the $(\mathrm{H})$-convergence in 2-metric spaces.

Theorem 5. Let $X$ be a 2-metric space, $\left\{X_{n}\right\}_{n \in \overline{\mathbb{N}}}$ a family of nonempty subsets of $X,\left\{T_{n}: X_{n} \rightarrow X\right\}_{n \in \overline{\mathbb{N}}}$ a family of mappings satisfying the property $(H)$ and such that $T_{\infty}$ is a $(\psi, \phi)$-weakly contractive mapping. If for any $n \in \overline{\mathbb{N}}, x_{n}$ is a fixed point of $T_{n}$ then the sequence $\left\{x_{n}\right\}_{n \in \mathbb{N}}$ converges to $x_{\infty}$.

Proof. By the property $(\mathrm{H})$, there exists a sequence $\left\{y_{n}\right\}$ in $X_{\infty}$ such that $\lim _{n \rightarrow \infty} \rho\left(x_{n}, y_{n}, a\right)=0$ and $\lim _{n \rightarrow \infty} \rho\left(T_{n} x_{n}, T_{\infty} y_{n}, a\right)=0$ for any $a \in X$. Hence for any $a \in X$,

$$
\begin{aligned}
\rho\left(x_{n}, x_{\infty}, a\right)= & \rho\left(T_{n} x_{n}, T_{\infty} x_{\infty}, a\right) \\
\leq & \rho\left(T_{n} x_{n}, T_{\infty} y_{n}, a\right) \\
& +\rho\left(T_{\infty} y_{n}, T_{\infty} x_{\infty}, a\right)+\rho\left(T_{n} x_{n}, T_{\infty} x_{\infty}, T_{\infty} y_{n}\right) .
\end{aligned}
$$

Since $\psi$ is increasing,

$$
\begin{aligned}
\psi\left(\rho\left(x_{n}, x_{\infty}, a\right)\right) \leq & \psi\left(\rho\left(T_{n} x_{n}, T_{\infty} y_{n}, a\right)\right. \\
& \left.+\rho\left(T_{\infty} y_{n}, T_{\infty} x_{\infty}, a\right)+\rho\left(T_{n} x_{n}, T_{\infty} x_{\infty}, T_{\infty} y_{n}\right)\right) .
\end{aligned}
$$

Now using the properties of $\psi$ and $\phi$ and making $n \rightarrow \infty$,

$$
\begin{aligned}
\lim _{n \rightarrow \infty} \psi\left(\rho\left(x_{n}, x_{\infty}, a\right)\right) \leq & \lim _{n \rightarrow \infty} \psi\left(\rho\left(T_{\infty} y_{n}, T_{\infty} x_{\infty}, a\right)\right) \\
\leq & \lim _{n \rightarrow \infty}\left[\psi\left(\rho\left(y_{n}, x_{\infty}, a\right)\right)-\phi\left(\rho\left(y_{n}, x_{\infty}, a\right)\right)\right] \\
\leq & \lim _{n \rightarrow \infty}\left[\psi\left(\rho\left(y_{n}, x_{\infty}, x_{n}\right)+\rho\left(y_{n}, x_{n}, a\right)+\rho\left(x_{n}, x_{\infty}, a\right)\right)\right. \\
& \left.\quad-\phi\left(\rho\left(y_{n}, x_{\infty}, x_{n}\right)+\rho\left(y_{n}, x_{n}, a\right)+\rho\left(x_{n}, x_{\infty}, a\right)\right)\right] \\
& =\lim _{n \rightarrow \infty} \psi\left(\rho\left(x_{n}, x_{\infty}, a\right)\right)-\lim _{n \rightarrow \infty} \phi\left(\rho\left(x_{n}, x_{\infty}, a\right)\right) .
\end{aligned}
$$


Thus

$$
\lim _{n \rightarrow \infty} \phi\left(\rho\left(x_{n}, x_{\infty}, a\right)\right) \leq 0
$$

By the property of $\phi$, we deduce that

$$
\lim _{n \rightarrow \infty} \rho\left(x_{n}, x_{\infty}, a\right)=0,
$$

and the conclusion follows.

Corollary 12 ([27, Theorem 3.4]). Let $X$ be a 2-metric space, $\left\{X_{n}\right\}_{n \in \overline{\mathbb{N}}}$ a sequence of nonempty subsets of $X,\left\{T_{n}: X_{n} \rightarrow X\right\}_{n \in \overline{\mathbb{N}}}$ a sequence of mappings satisfying the property $(H)$ and such that $T_{\infty}$ is a $k$-contraction. If for any $n \in \overline{\mathbb{N}}, x_{n}$ is a fixed point of $T_{n}$ then the sequence $\left\{x_{n}\right\}_{n \in \mathbb{N}}$ converges to $x_{\infty}$.

Proof. This comes from Theorem 5, when $\psi(t)=t$ and $\phi(t)=(1-k) t$ and $k \in(0,1)$.

Corollary 13 ([25, Theorem 4.5]). Corollary 12 with $k$-contraction replaced by nonlinear contraction.

Acknowledgement. The authors are indebted to the referee for bringing in some new information which has been useful for the improvement of the paper.

\section{REFERENCES}

[1] S. P. Acharya, Convergence of a sequence of fixed points in a uniform space, Mat. Vesnik. 13(28)(1976), 131-141.

[2] Ya. I. Alber, and S. Guerre-Delabriere, Principle of weakly contractive maps in Hilbert spaces. New results in operator theory and its applications, 7-22, Oper. Theory Adv. Appl., 98, Birkhäuser, Basel, 1997.

[3] V. G. Angelov, A continuous dependence of fixed points of $\phi$-contractive mappings in uniform spaces, Archivum Mathematicum (Brno) 28(1992), (3-4), 155-162.

[4] L. Barbet and K. Nachi, Convergence des points fixes de $k$-contractions (Convergence of fixed points of $k$-contractions), (2006) Preprint, University of Pau.

[5] L. Barbet and K. Nachi, Sequences of contractions and convergence of fixed points, Monografias del Seminario Matemático García de Galdeano 33(2006), 51-58.

[6] F. F. Bonsall, Lectures on Some Fixed Point Theorems of Functional Analysis, Tata Institute of Fundamental Research, Bombay 1962.

[7] R. K. Bose and M. K. Roychowdhury, Fixed point theorems for generalized weakly contractive mappings, Surv. Math. Appl. 4 (2009), 215-238.

[8] D. W. Boyd and J. S. W. Wong, On nonlinear contractions, Proc. Amer. Math. Soc. 20(1969), 458-464.

[9] B. S. Choudhury, P. Konar, B. E. Rhoades and N. Metiya, Fixed point theorems for generalized weakly contractive mappings, Nonlinear Anal. 74(6)(2011), 2116-2126.

[10] J. Dugundji and A. Granas, Weakly contractive maps and elementary domain invariance theorem, Bull. Greek. Math. Soc. 19(1978), 141-151.

[11] P. N. Dutta and B. S. Choudhury, A generalisation of contraction principle in metric spaces, Fixed Point Theory Appl. 2008, Art. ID 406368, 8 pp. 
[12] S. Gähler, 2-metrische Räume und ihre topologische Struktur, Math. Nachr. 26(1963/64), 115-148.

[13] K. Iséki, Mathematics in 2-normed spaces, Math. Sem. Notes Kobe Univ. 4(1974), 161-174.

[14] K. Iséki, Fixed point theorem in 2 -metric spaces, Math. Sem. Notes Kobe Univ. 3(1975), 133-136.

[15] K. Iséki, Mathematics in 2-metric space, Bull. Korean Math. Soc. 132(1976), 127135.

[16] K. Iséki, P. L. Sharma and B. K. Sharma, Contraction type mapping on 2-metric space, Math. Japonicae 21 (1976), 67-70.

[17] J. Jachymski: Equivalent conditions for generalized contractions on (ordered) metric spaces, Nonlinear Anal. 74 (2011) 768-774.

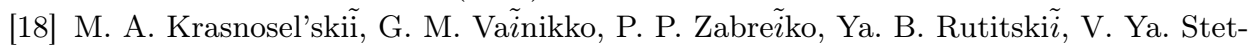
senko: Approximate solution of operator equations, Wolters- Noordhoff Publishing, Groningen, (1972).

[19] S. N. Lal and A. K. Singh, An analogue of Banach's contraction principle for 2-metric spaces, Bull. Austral. Math. Soc. 18(1978), 137-143.

[20] S. N. Mishra, On sequences of mappings and fixed points, Rend. Sem. Mat. Univers. Politecn. Torino 34(1976), 405-410.

[21] S. N. Mishra, On sequences of mappings and fixed points II, Indian J. Pure Appl. Math. 10(1979), 699-703.

[22] S. N. Mishra, On common fixed points of multimappings in uniform spaces, Indian J. Pure Appl. Math. 13 (5)(1991), 606-608.

[23] S. N. Mishra and A. K. Kalinde, On certain stability results of Barbet and Nachi, Fixed Point Theory 12 (1)(2011), 137-144.

[24] S. N. Mishra and Rajendra Pant, Sequences of $\phi$-contractions and stability of fixed Points, Indian J. Math. 54(2)(2012), 211-223.

[25] S. N. Mishra, Rajendra Pant and R. Panicker, Sequences of nonlinear contractions and stability of fixed points, Advances in Fixed Point Theory, 2 (2012), No. 3, 298 312 .

[26] S. N. Mishra, Rajendra Pant and R. Panicker, Sequences of $(\psi, \phi)$ - weakly contractive mappings and stability of fixed points, Int. Journal of Math. Analysis, 7 (22)(2013),1085-1096.

[27] S. N. Mishra, S. L. Singh and Rajendra Pant, Some new results on stability of fixed points, Chaos, Solitons \& Fractals, 45 (2012) 1012-1016.

[28] S. N. Mishra, S. L. Singh, Rajendra Pant and S. Stofile, Some new notions of convergence and stability of common fixed points in 2-metric spaces, Advances in Fixed Point Theory,2(2012), No. 1, 64-78.

[29] S. N. Mishra, S. L. Singh and S. Stofile, Stability of common fixed points in uniform spaces, Fixed Point Theory and Applications, 2011:37, 1-8.

[30] S. B. Nadler, Jr., Sequences of contractions and fixed points, Pacific J. Math. 27(1968), 579-585.

[31] S. V. R. Naidu and J. Rajendra Prasad, Fixed points theorems in 2-metric spaces, Indian J. Pure Appl. Math. 17 (8)(1986), 974-993.

[32] B. E. Rhoades, Contraction type mappings on a 2 -metric space, Math. Nachr. 91(1979), 151-156.

[33] B. E. Rhoades, Some theorems on weakly contractive maps. Nonlinear Anal. 47 (2001), no. $4,2683-2693$. 
[34] S. L. Singh, A note on the convergence of a pair of sequences of mappings, Arch. Math. 15(1)(1979), 47-52.

[35] S. L. Singh and B. Ram, A note on the convergence of sequences of mappings and their common fixed points in a 2-metric space, Math. Sem. Not. 9(1981), 181-185.

[36] S. L. Singh and B. Ram, A note on the convergence of sequences of mappings and their common fixed points in a 2-metric space II, J. Univ. Kuwait Sci. 10(1993), 31-35.

[37] S. P. Singh, On a theorem of Sonnenshein, Bull. de l'Acadé mie Royale de Belgique 3(1969), 413-414.

[38] S. P. Singh and W. Russel, A note on a sequence of contraction mappings, Can. Math. Bull. 12(1969), 513-516.

[39] J. Sonnenshein, Opérateurs de même coefficient de contraction, Bulletin de l'Académie Royale de Belgique 52(1966), 1078-1082.

[40] M. Taskovic, A monotone principle of fixed points, Proc. Amer. Math. Soc. 94(1985), 427-432.

[41] M. Taskovic, Some new principles in fixed point theory, Math. Japonica 35(4)(1990), 645-666.

\author{
S.N. Mishra \\ Department of Mathematics \\ Walter Sisulu University \\ МтнатHA 5117 \\ South Africa \\ E-mail address: swaminathmishra3@gmail.com
}

\section{RAJENDRA PANT}

Department of Mathematics

Visvesvaraya National Institute of Technology

NAGPUR 440010

INDIA

E-mail address: pant.rajendra@gmail.com

\author{
R. PANicker \\ Department of Mathematics \\ Walter Sisulu University \\ MTHATHA 5117 \\ South Africa \\ Secondary address(Research Scholar) \\ Department of Mathematics \\ Rhodes UNIVERSITY \\ Grahamstown 6140 \\ South AfricA \\ E-mail address: rpanicker@wsu.ac.za
}

